\title{
Dynamic brainstem and somatosensory cortical excitability during migraine cycles
}

\author{
Fu-Jung Hsiao ${ }^{1}$, Wei-Ta Chen ${ }^{1,2,3,4^{*}} \mathbb{0}$, Li-Ling Hope Pan ${ }^{1}$, Hung-Yu Liu ${ }^{2,3}$, Yen-Feng Wang ${ }^{2,3}$, Shih-Pin Chen ${ }^{1,2,3}$, \\ Kuan-Lin Lai ${ }^{2,3}$, Gianluca Coppola ${ }^{5}$ and Shuu-Jiun Wang ${ }^{1,2,3^{*}}$
}

\begin{abstract}
Background: Migraine has complex pathophysiological characteristics and episodic attacks. To decipher the cyclic neurophysiological features of migraine attacks, in this study, we compared neuronal excitability in the brainstem and primary somatosensory (S1) region between migraine phases for 30 consecutive days in two patients with episodic migraine.
\end{abstract}

Methods: Both patients underwent EEG recording of event-related potentials with the somatosensory and pairedpulse paradigms for 30 consecutive days. The migraine cycle was divided into the following phases: $24-48 \mathrm{~h}$ before headache onset (Pre2), within 24h before headache onset (Pre1), during the migraine attack (Ictal), within $24 \mathrm{~h}$ after headache offset (Post 1), and the interval of ' $48 \mathrm{~h}$ between the last and next headache phase (Interictal). The normalised current intensity in the brainstem and $\mathrm{S} 1$ and gating ratio in the S1 were recorded and examined.

Results: Six migraine cycles (three for each patient) were analysed. In both patients, the somatosensory excitability in the brainstem (peaking at 12-14 ms after stimulation) and S1 (peaking at 18-19 ms after stimulation) peaked in the Pre1 phase. The $\mathrm{S} 1$ inhibitory capability was higher in the Ictal phase than in the Pre1 phase.

Conclusion: This study demonstrates that migraine is a cyclic excitatory disorder and that the neural substrates involved include the somatosensory system, starting in the brainstem and spanning subsequently to the S1 before the migraine occurs. Further investigations with larger sample sizes are warranted.

Keywords: Brainstem, Primary somatosensory cortex (S1), Migraine cycle, Excitability, Inhibition

\section{Introduction}

Migraine is the most prevalent neurological disorder, and it is the second most debilitating disease worldwide [1]. It has complex pathophysiological characteristics and episodic attacks of moderate to severe headache accompanied by nausea, vomiting, or hypersensitivity to sensory stimuli such as light, sound, and odour. Mounting evidence suggests that migraine symptoms are determined by complex interactions among genetic, environmental, hormonal, and other endogenous factors [2, 3]. Although the underlying neuropathological mechanism remains

\footnotetext{
*Correspondence: wtchen@vghtpe.gov.tw; sjwang@vghtpe.gov.tw

${ }^{3}$ Department of Neurology, Neurological Institute, Taipei Veterans General Hospital, 201, Shihpai Rd Sec 2, Taipei 112, Taiwan

Full list of author information is available at the end of the article
}

unclear, a fundamental imbalance of neuronal excitability (i.e. neuronal dysexcitability) may play a pivotal role [4]. In brain excitability studies, functional changes during migraine attacks have included hyperresponsivity to repeated sensory stimuli, altered recruitment of neuronal networks, and impaired habituation [4-9]. However, research results on excitability have differed because of diverse intervals from migraine attacks among study populations $[9,10]$. To resolve this challenge, brain excitability dynamics should be recorded throughout entire migraine cycles.

In pioneering migraine studies, Schulte and colleagues used 30-day functional MRI (fMRI) after trigeminal nociceptive stimulation or in the resting state to investigate prodromal functional changes in neural activity and functional coupling within the brainstem, hypothalamus, 
nucleus accumbens, amygdala, hippocampus, and visual cortex [11-13]. Hypothalamus-brainstem connectivity has been implicated as a driver of migraine attacks [11], and enhanced brainstem activation may be a marker of a migraine attack (i.e. cyclic activation) [11-13]. These findings implicate the dysfunction of the brainstem and its relevant network in migraine, suggesting that such neurological changes led to this complex brain disorder [14]. However, fMRI does not directly measure neural activity $[15,16]$, and blood oxygen level-dependent signals are limited by low temporal resolution. Additionally, continuous trigeminal nociceptive stimulation could trigger adverse plastic adaptive changes in several brain areas, which may have biased the results [17]. Therefore, somatosensory-evoked potentials (SSEPs) may be more appropriate for examining brain excitability dynamics during the entirety of a migraine cycle because they exhibit superior temporal resolution, can be recorded noninvasively and with innocuous stimulation, and are direct reflection of neural activity [4]. Moreover, impaired brainstem function even in the interictal period was noted in one EEG migraine study [18].

Central sensitisation of the primary somatosensory cortex (S1) and impaired response habituation to repetitive afferent stimuli are also crucial neurophysiological occurrences in migraine $[4,19]$. In our recent studies $[6$, 20], S1 gating, a habituation-related but more basic protective mechanism against brain sensory overload, was altered in patients with migraine, and interictal inhibitory function (i.e. gating ratio) was linked to migraine severity. However, to determine the cyclic characteristics of migraines, S1 inhibition throughout the migraine cycle should be analysed longitudinally.

As mentioned, neuronal excitability and inhibition are neurophysiological indicators in migraines, especially in the brainstem and S1. To determine the cyclic neurophysiological features of migraines, this study examined the evoked activity of the brainstem and S1 region in various migraine phases; the evoked activity was recorded each morning for 30 consecutive days in patients with episodic migraine (EM) by using EEG techniques with nonpainful somatosensory stimulation.

\section{Methods}

\section{Participants}

Two female patients (aged 26 and 32 years) who were diagnosed with EM without aura according to the third edition of the International Classification of Headache Disorders [21], were recruited from the Headache Clinic of Taipei Veterans General Hospital. Neither patient had a history of systemic or major neurological disease. Both patients had normal results on physical and neurological examinations, and both were right handed. Both willingly joined this study for a 30-day period and provided signed informed consent. Both completed a daily headache diary and EEG recordings. Both were asked to refrain from acute medication consumption for at least $16 \mathrm{~h}$ before the EEG recordings. They did not take any preventive medication, neither during the studying period nor before the study. The hospital's institutional review board approved the study protocol (VGHTPE: IRB 2019-07-001B).

\section{Study design}

During the 30-day period, headache status (headache or no headache), headache characteristics (pain rating and pain location), menstrual cycle status, use of analgesic medications, and presence of premonitory and other migraine-associated symptoms were assessed daily. The daily levels of pain intensity, anxiety, depression, and stress were subjectively rated on a visual analogue scale anchored at 0 and 10 . Moreover, cutaneous allodynia was evaluated before the study using a 17-item questionnaire as reported in our related work [22]. Additionally, each participant underwent EEG recording of event-related potentials with standardised electrical somatosensory stimulation between 10:00 a.m. and 12:00 p.m. each day for 30 days. The somatosensory and paired-pulse paradigms were employed. After the 30 days of recordings, pain-free T1 structural images were acquired for further EEG source analysis.

For a migraine cycle to be analysed, it must feature a headache attack preceded by at least 3 pain-free days, not coincide with another migraine cycle (to exclude possible postdromal effects), and have acceptable EEG data recorded without any technical problems. Thus, the number of analysed attacks could differ from the number of observed attacks. We divided the peri-ictal, ictal, and interictal periods into phases according to their relative time as follows [12, 21]: $24-48 \mathrm{~h}$ before headache onset was Pre2, within 24h before headache onset was Pre1, during the migraine attack was Ictal, within $24 \mathrm{~h}$ after headache offset was Post1, and the period ' $48 \mathrm{~h}$ from the last and next headache phase was Interictal. Any day with a tension-type headache (TTH) attack during the interictal phase was excluded from analysis.

\section{EEG recording and analysis}

Scalp EEG data were collected from an EEG cap housing a 64-electrode BrainVision actiCAP system (Brain Products $\mathrm{GmbH}$, Munich, Germany) that covered the entire brain according to the extended International 10-20 system [23]. Active circuits for impedance conversion were integrated into the slim actiCAP electrodes, enabling high signal quality at higher impedances than conventional passive electrodes allow. The electrodes were referenced online to an electrode placed on the Fz plane, and 
a common ground connection was established at the $\mathrm{FPz}$ site. The EEG signals were amplified and digitised using a BrainAmp DC amplifier (Brain Products GmbH, Munich, Germany) linked to Brain Vision Recorder software (version 2.1, Brain Products GmbH, Munich, Germany).

Somatosensory-evoked and paired-pulse stimulation was delivered to each patient during the daily EEG recordings. For the two stimulation tests, a Digitimer DS7A device (Digitimer, Welwyn Garden City, Hertfordshire, UK) with constant-current square-wave pulses (0.2-ms width, proximal cathode) was used; the intensity of electrical stimulation of the right median nerve at the wrist was twice the subjective sensory threshold, and no pain response or visible twitching of the flexor digitorum superficialis was elicited. The patients were comfortably seated on a chair in an illuminated room and asked to remain awake with their eyes closed. Evoked brain activity was continually recorded at a digital sampling rate of $1000 \mathrm{~Hz}$. For the somatosensory test, electrical stimulation was delivered at $4 \mathrm{~Hz} / \mathrm{s}$ to collect 1000 samples of SSEPs, including a prestimulus baseline of $50 \mathrm{~ms}$ and poststimulus measurement of $100 \mathrm{~ms}$, for a sufficient number of samples to reliably determine average brainstem responses [24]. For the paired-pulse paradigm, the stimulation comprised paired pulses applied to the right median nerve with an interstimulus interval of $500 \mathrm{~ms}$ and an interpair interval of $8 \mathrm{~s}$ [25]. The length of each recorded trial, except for the prestimulus baseline of $50 \mathrm{~ms}$, was $150 \mathrm{~ms}$. At least 100 artefact-free responses to the first and second pulses of the paired stimuli (hereafter referenced as 'first response' and 'second response', respectively) were recorded. Notably, to avoid fatigue, a 10-min break was granted between the two tests.

Distributed current source modelling of EEG data was performed using depth-weighted minimum norm estimates (MNEs) [6, 20, 26], which accurately resolve source localisation, even for deep generators $[27,28]$. The neuronal dynamics of cortical and subcortical sources were determined using a deep brain model that describes the signal patterns generated by a unit dipole, realistically distributing current dipoles over the neocortex and subcortical structures [28]. This forward model uses the symmetric boundary element method [29], which provides more accurate results than spherical models provide. Structural brain imaging was performed using a 3 T MR system (Magnetom Tim Trio; Siemens, Malvern, PA, USA) with the following parameters: repetition time, $9.4 \mathrm{~ms}$; echo time, $4 \mathrm{~ms}$; recording matrix, $256 \times 256$ pixels; field of view, $256 \mathrm{~mm}$; and slice thickness, $1 \mathrm{~mm}$. The shapes of surfaces separating the scalp, skull, and brain compartments were identified using FreeSurfer 7.0 software (Harvard, Cambridge, MA, USA), which was also used for the subcortical segmentation of brain volume.
The inverse operator of MNE analysis was used to estimate the distribution of the current sources that account for the data recorded at the electrodes. The aforementioned analysis resulted in distributed and dynamic brain activation that could be mapped onto the reconstructed surface and volume for each patient; consequently, the time-varying current intensity could be extracted from the brainstem ('volume scout' function) and S1 ('surface scout' function). In the somatosensory-evoked task, the peak current intensity in the brainstem at $12-13 \mathrm{~ms}$ and S1 at $17-18 \mathrm{~ms}$ was obtained [18]. To highlight the components from the subcortical generators, the current density values were transformed into $z$ scores which represented the number of standard deviations from the baseline level. To compare the dynamic neural excitability of different migraine attacks, the current density in each phase of the migraine cycle (Pre2, Pre1, Ictal, Post1, and Interictal phases) was also normalised in relation to the ictal day (Ictal) of the cycle. For the paired-pulse task, the peak S1 current density was obtained, and the gating ratio was subsequently determined (current density of second response/current density of first response) [6, $20,25]$. Data analysis was performed using Brainstorm software [30], which has been partially described in our previous papers $[6,20]$.

\section{Statistical analysis}

The normalised current intensity and gating ratio were obtained from the two patients' selected migraine cycles and examined by phase (Pre2, Pre1, Ictal, Post1, and Interictal). Nonparametric tests were applied because the data were not normally distributed. Specifically, the Kruskal-Wallis test was initially used to compare phases. Second, the Wilcoxon signed-rank test was used to compare the normalised current intensity and gating ratio of the Pre 2 and Pre1, Pre1 and Ictal, and Ictal and Post1 phases. Finally, the Mann-Whitney test was used to compare the Interictal phase with the other phases within the cycle. All tests were two tailed, and the significance level was $P<0.05$. Bonferroni correction was applied for multiple comparisons.

\section{Results}

\section{Symptom description}

The headache conditions and subjective psychometric scores of the two patients during the 30-day period are illustrated in Fig. 1. Patient 1 was a woman aged 32 years with a 20-year history of EM. During the 30-day recording period, three of her migraine cycles (shaded in pink in Fig. 1) qualified for analysis. In the preictal phase, she reported no aura but had prodromal symptoms of fatigue and yawning. Her migraine attacks were unilateral but not fixed to the right or left side. On days 20 and 21, 


\section{Patient \#1}

Days
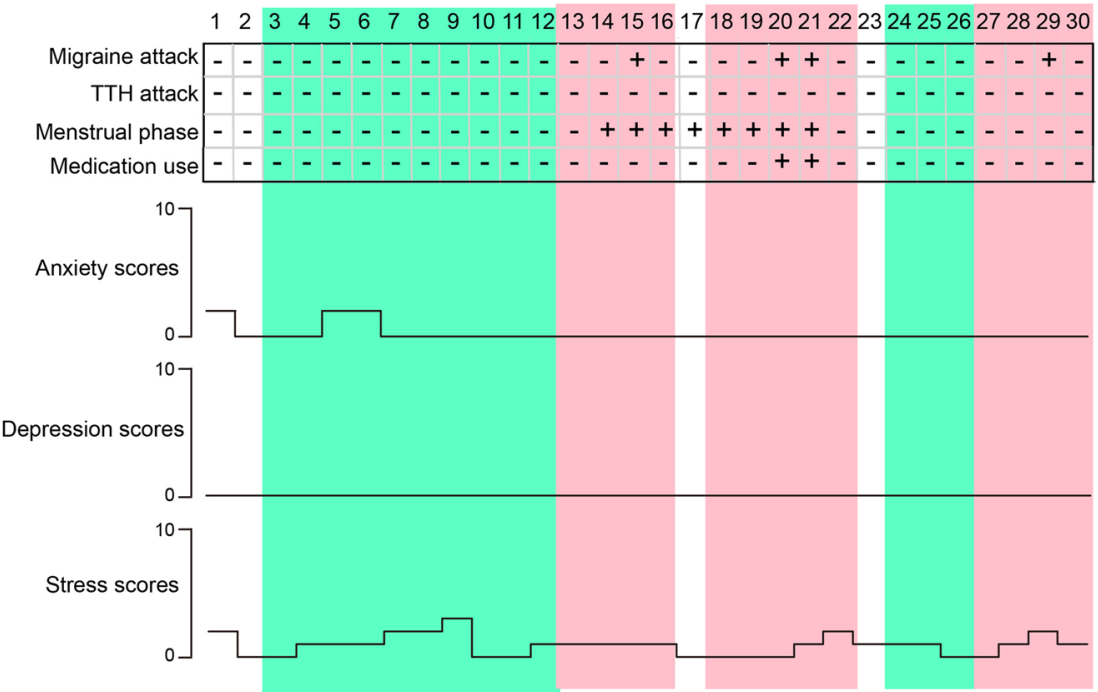

\section{Patient \#2}

\section{Days}

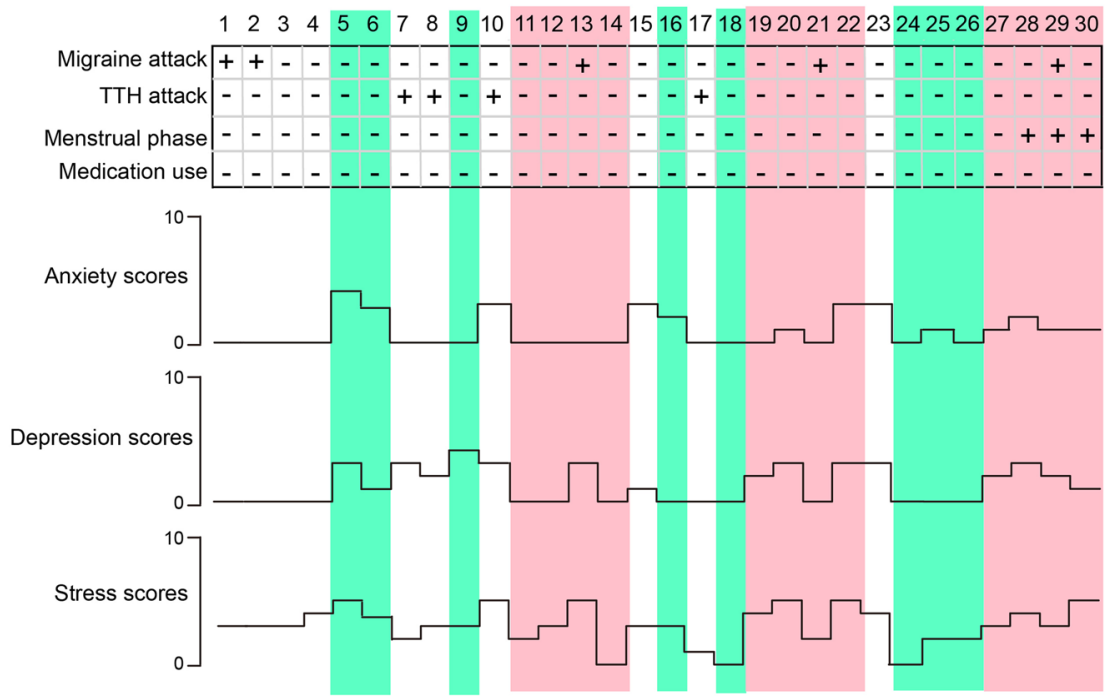

Fig. 1 Headache attacks and clinical scores during 30-day period. Headache attack, menstrual phase, medication use, and psychometric scores within consecutive 30 days in two patients with EM. Days shaded in pink are parts of migraine cycles. Days shaded in aquamarine are in the pain-free interictal period. TTH, tension-type headache

$500 \mathrm{mg}$ of acetaminophen were administered after EEG recording. Patient 2 was a 26-year-old woman with a 2-year history of EM. In the 30-day period, three of her migraine cycles (shaded in pink in Fig. 1) met the criteria. During the preictal phase, neither aura nor prodromal symptoms were reported. The patient's headaches were unilateral but with no fixed side. Headache attacks on days $7,8,10$, and 17 were considered TTHs because of their symptoms. For these two patients, a total of 21 days (shaded in green) were considered parts of interictal periods, but only 20 days of data were eligible for interictal analysis because a technical problem with the EEG device occurred on day 16 for patient 2. Both patients were free of cutaneous allodynia according to the baseline evaluation.

\section{Somatosensory-evoked responses during the migraine cycle}

In response to somatosensory stimulation, the superimposed waveforms of evoked potentials from all electrodes 
are depicted in the upper panels of Fig. 2A and Fig. 2C for the preictal period for patient 1 (day 20) and patient 2 (day 12), respectively. Three prominent components peaked at 12 (or 13), 18, and $24 \mathrm{~ms}$, and their corresponding topographies (lower panels of Fig. 2A and Fig. 2C) indicate subcortical and contralateral parietal activation. Figure $2 \mathrm{~B}$ and $\mathrm{D}$ present the neural activation at $9-18,18$, and $24 \mathrm{~ms}$ mapped onto individual brain images. For both patients, the early component (12 or $13 \mathrm{~ms}$ ) originated in the brainstem, and the later components (18 and $24 \mathrm{~ms}$ ) originated in the S1.

The dynamic current intensity ( $z$ score) of brainstem activation for -50 to $100 \mathrm{~ms}$ during one migraine cycle is presented in Fig. 3 for patient 1 (left) and patient 2 (right). The fluctuating brainstem activation was observed during the migraine cycle and the peak intensity of a clear

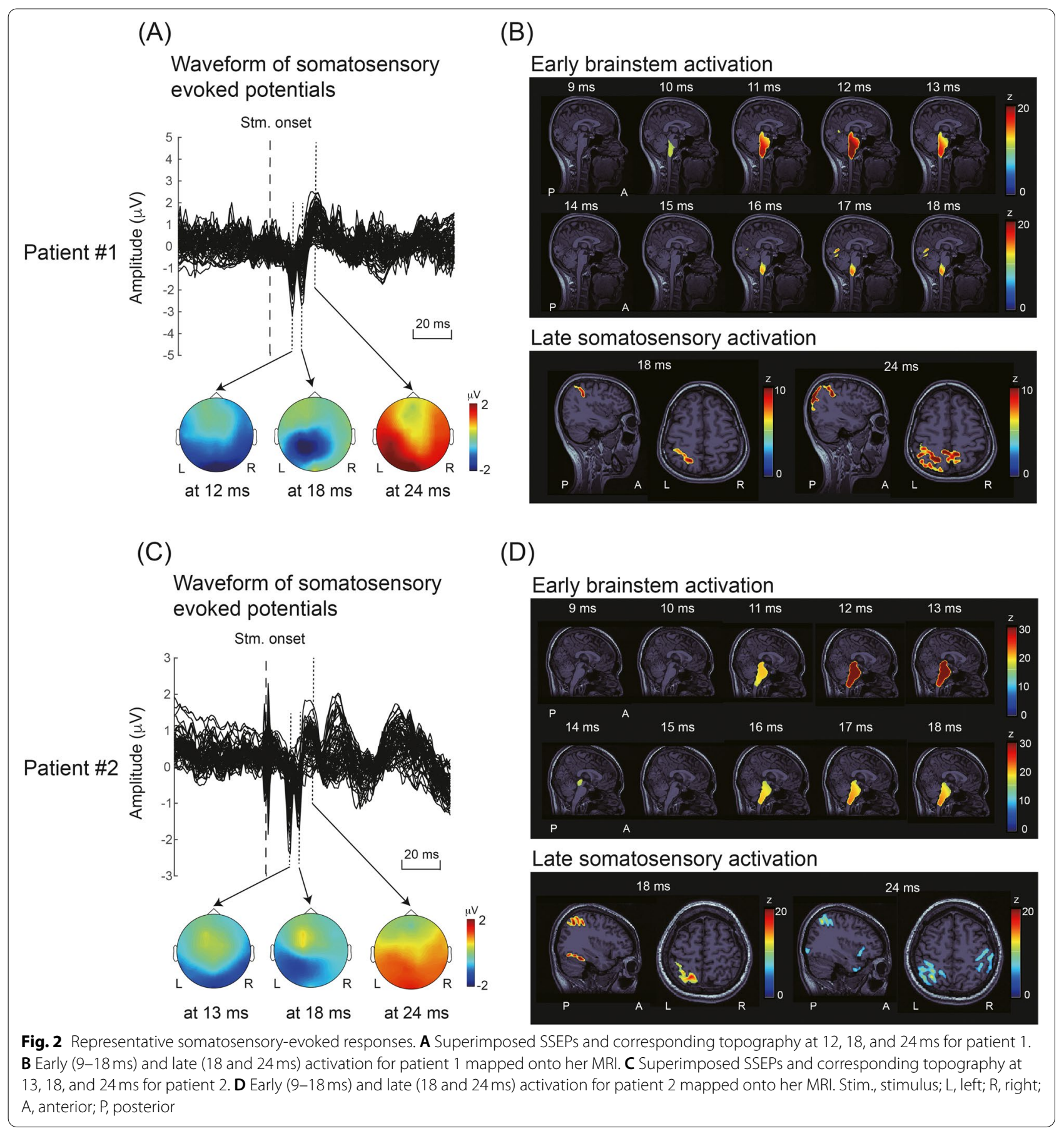




\section{Patient \#1}
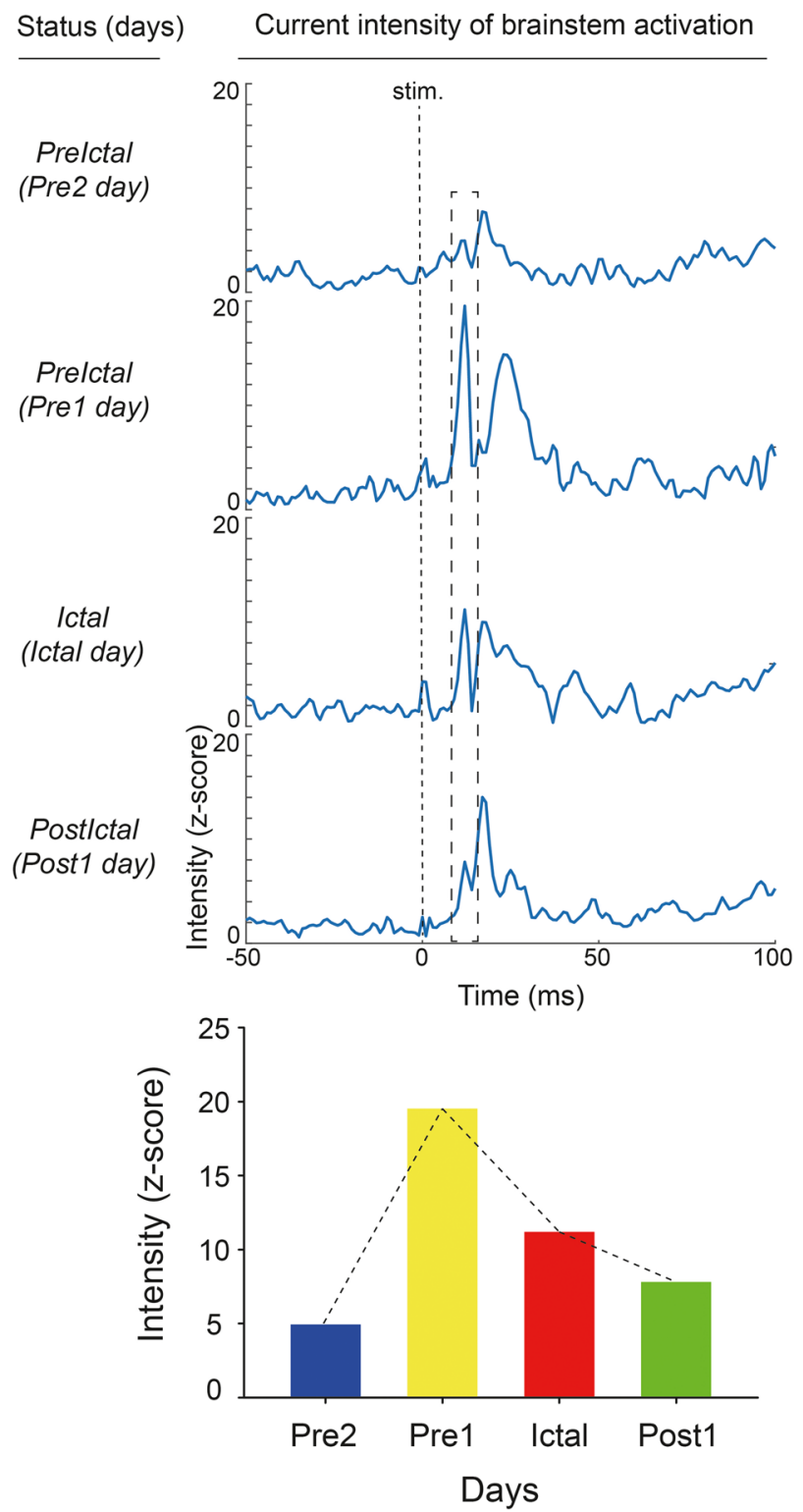

Patient \#2
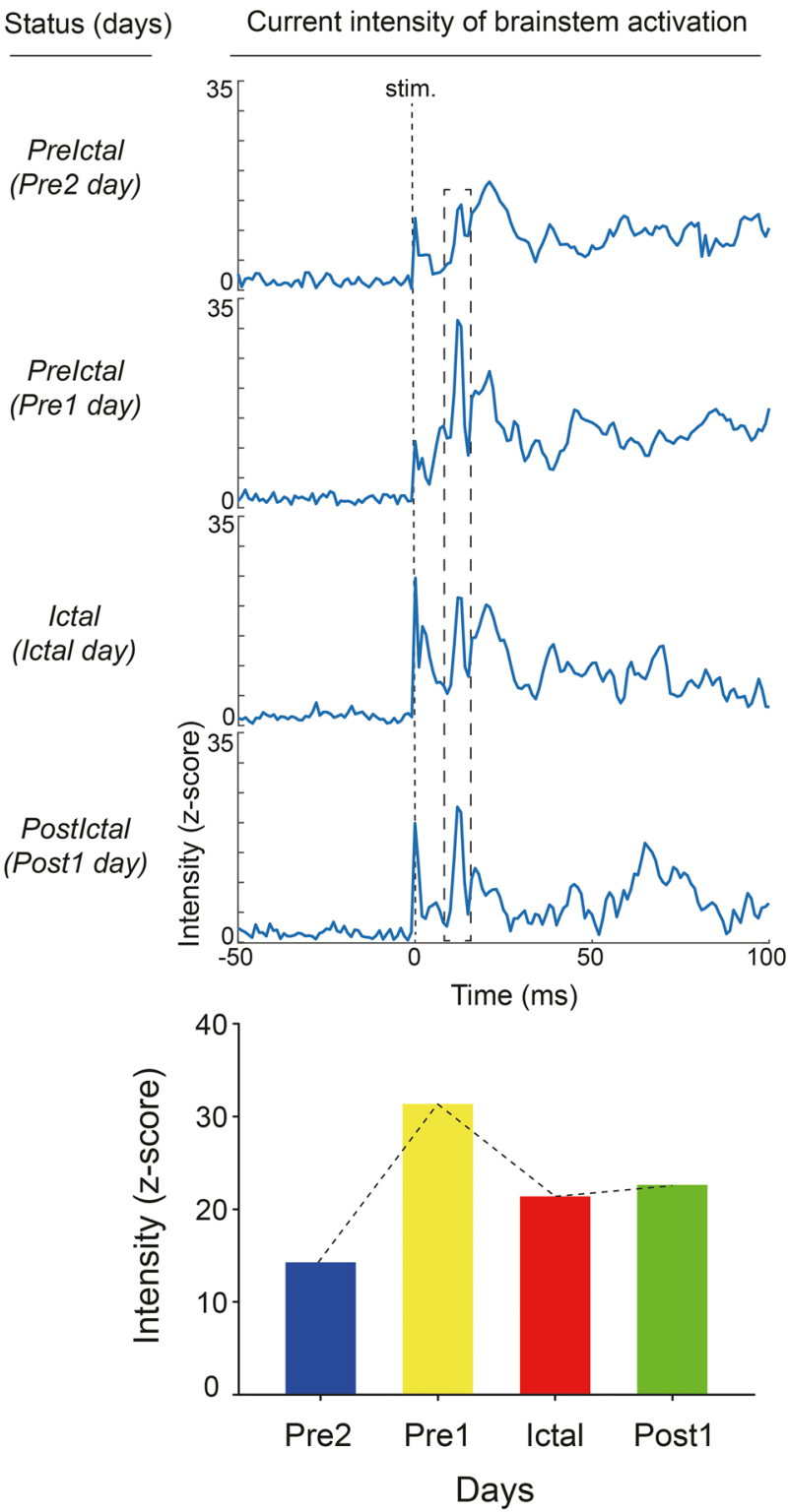

Fig. 3 Evoked brainstem activation during migraine cycle. Top: Brainstem activation over time ( -50 to $100 \mathrm{~ms}$ ) during a representative migraine cycle for patient 1 and patient 2. Bottom: Peak brainstem activation during migraine cycle. Pre2, 24-48 h before headache onset; Pre1, within 24h before headache onset; Ictal, during migraine attack; Post1, within $24 \mathrm{~h}$ after headache offset. Stim., stimulus onset

component at $12-14 \mathrm{~ms}$ was obtained (lower part of Fig. 3), demonstrating the association between brainstem activation and the migraine cycle. Specifically, the largest brainstem activation in both patients was noted in the preictal phase on the Pre1 day. Additionally, the S1 activation indicated dynamic current intensity during one migraine cycle in both patients (Fig. 4). The peak intensity of S1 activation was obtained at $18-19 \mathrm{~ms}$, and S1 activation fluctuated within the migraine cycle. Similar to brainstem activation, $\mathrm{S} 1$ activation was the strongest in the preictal phase (Pre1).

The normalised current intensity in the brainstem was compared between phases of the same migraine cycle ( $n=6$ for each phase) and each day of the migraine cycle and interictal period ( $n=20$; Fig. 5$)$. A significant difference in normalised current intensity 
Patient \#1
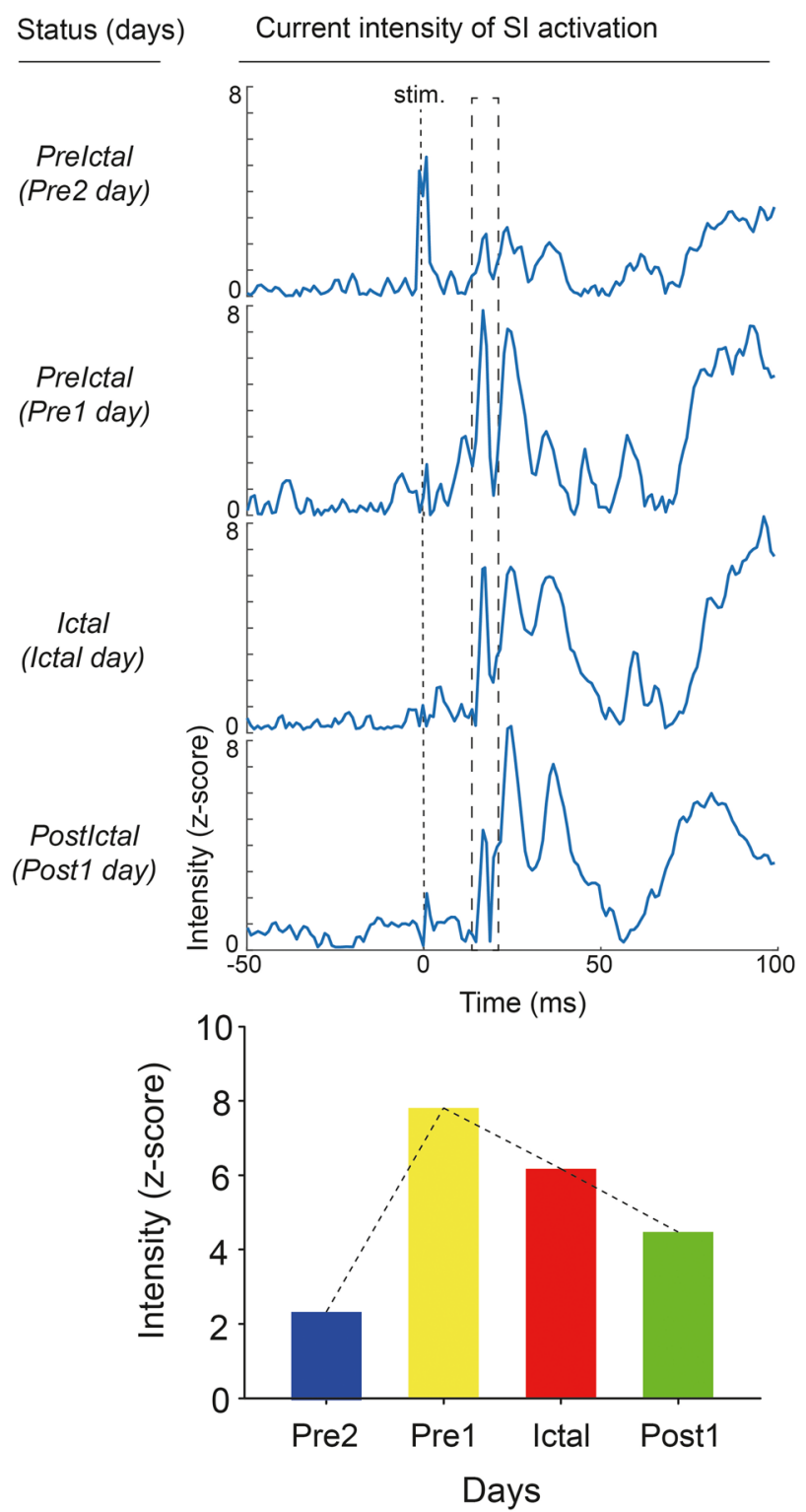

Patient \#2
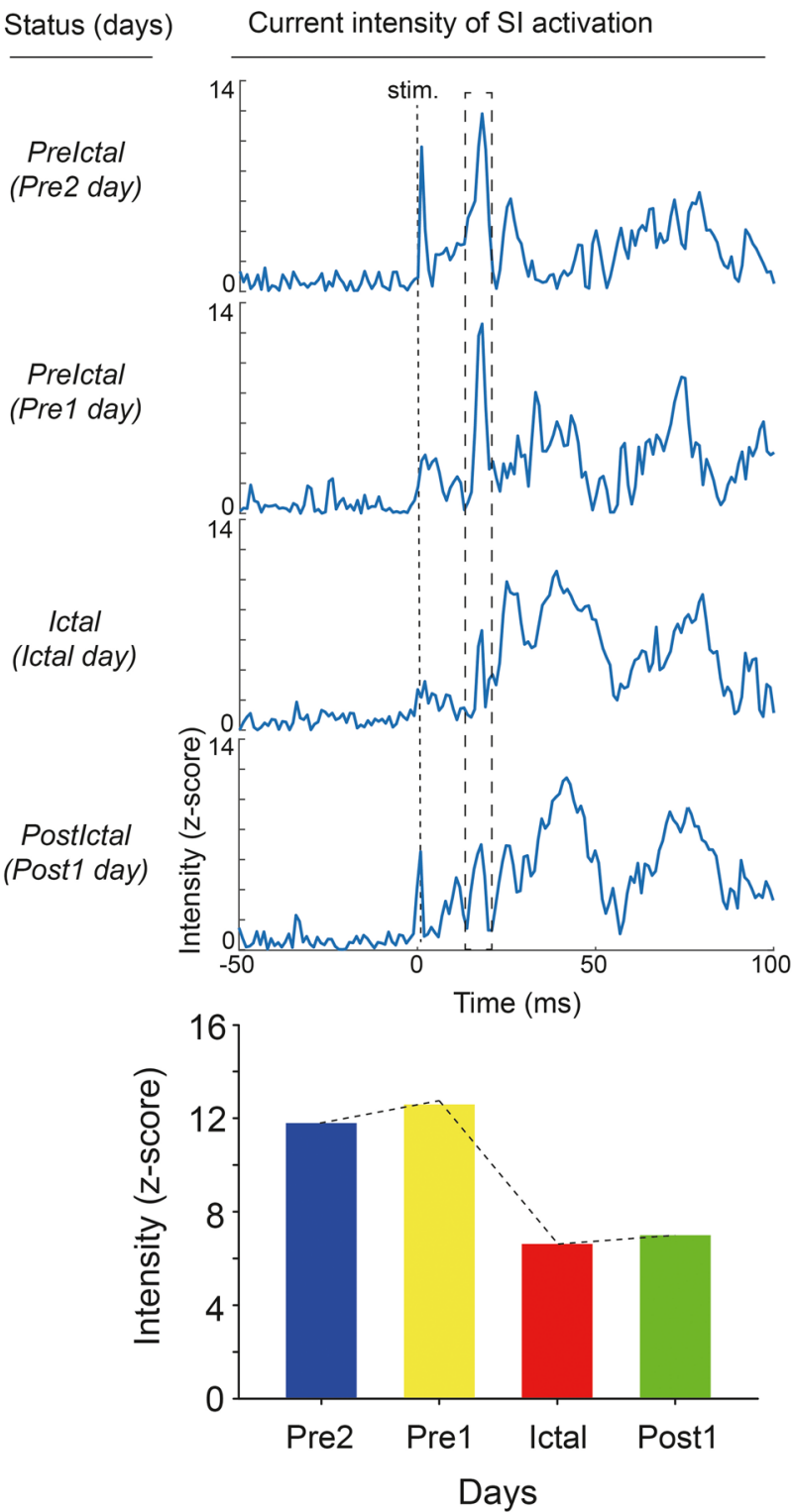

Fig. 4 Evoked current intensity of S1 activation during migraine cycle. Top: S1 current intensity over time ( -50 to 100 ms) within one representative migraine cycle for patient 1 and patient 2. Bottom: Peak S1 activation during migraine cycle. S1, primary somatosensory cortex; Pre2, 24-48 h before headache onset; Pre1, within $24 \mathrm{~h}$ after headache onset; Ictal, during migraine attack; Post1, within $24 \mathrm{~h}$ after headache offset

was observed between phases $\left(x^{2}=19.5, P=0.001\right)$. The Wilcoxon signed-rank test indicated a significant increase in brainstem current intensity from Pre2 to Pre1 $(z=2.2, P=0.028)$ and significant decrease from Pre1 to Ictal $(z=-1.99, P=0.046)$. Additionally, the normalised brainstem current intensity in the interictal period was lower than that in the Pre1 $(z=-3.1$, corrected $P=0.004)$ and Ictal $(z=-3.57$, corrected $P<0.001)$ phases. In the comparison of the
S1 activation on days within the migraine cycle and the interictal period (Fig. 6), significant differences in normalised current intensity were also observed between distinct days $\left(\chi^{2}=13.1, P=0.011\right)$. Specifically, a significant increase from Pre2 to Pre1 and a significant decrease from Pre1 to Ictal were noted in the S1 intensity (both $P<0.05$ ); moreover, the intensity in the interictal period was lower than it was in Pre1 $(z=-2.58$, corrected $P=0.04$ ). 


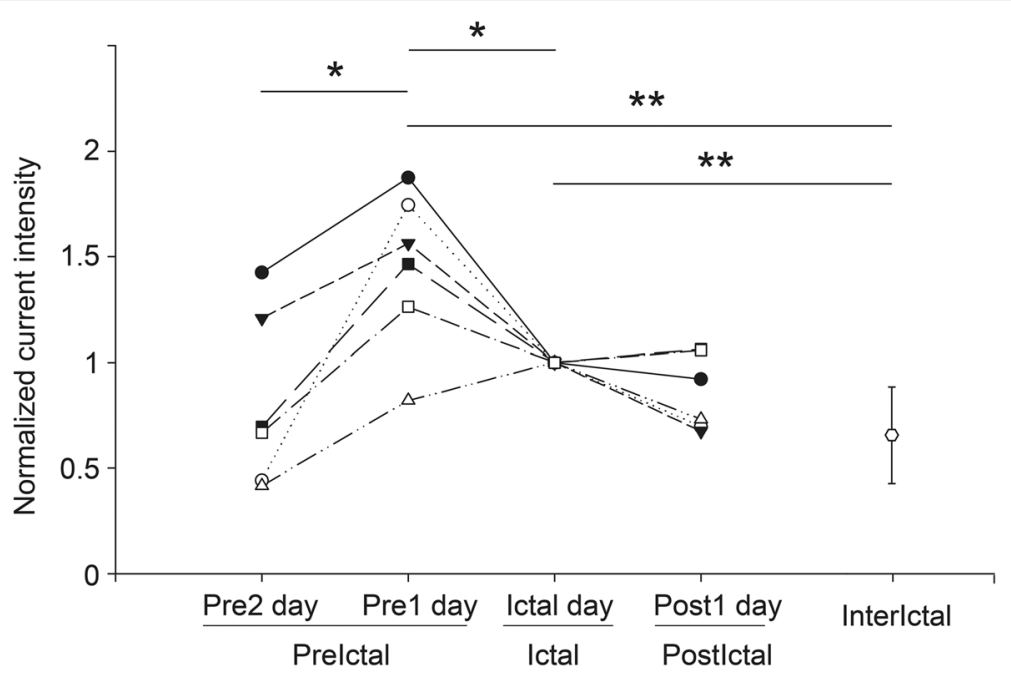

Fig. 5 Brainstem activation during migraine cycle. Differences in normalised peak brainstem intensity among migraine cycles $(n=6)$ and migraine phases (Pre2, Pre1, Ictal, Post1, and Interictal). ${ }^{*}, P<0.05 ;{ }^{*}, P<0.01 ;$ Pre2, 24-48 h before headache onset; Pre1, within $24 \mathrm{~h}$ before headache onset; Ictal, during migraine attack; Post 1 , within $24 \mathrm{~h}$ after headache offset; Interictal, the period ' $48 \mathrm{~h}$ between the last and next headache phase

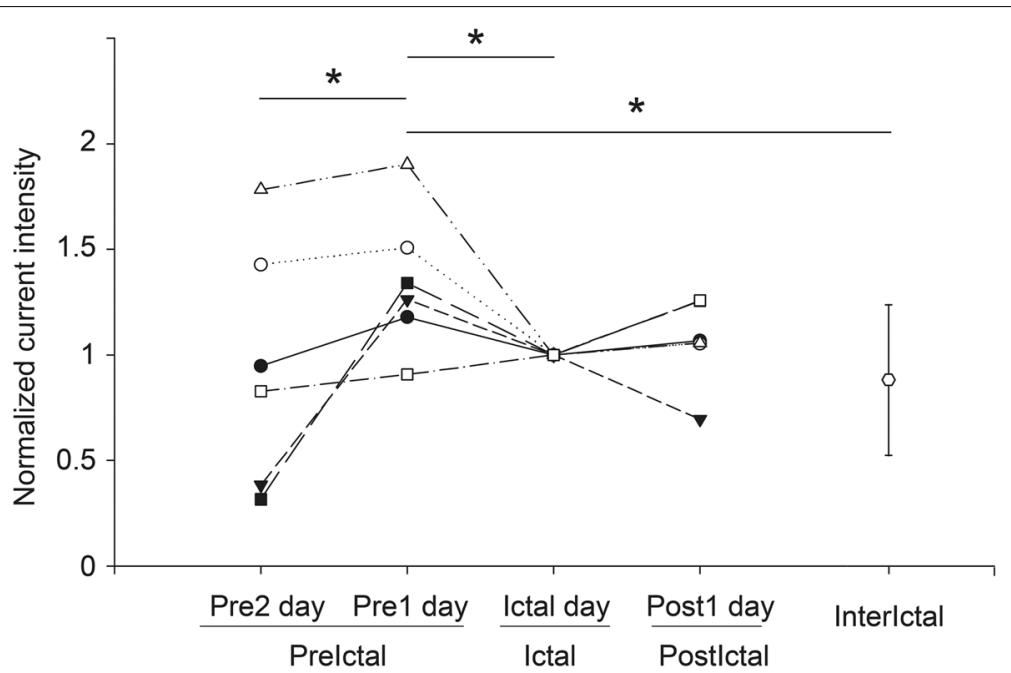

Fig. 6 S1 activation during migraine cycle. Differences in normalised peak S1 intensity among migraine cycles $(n=6)$ and migraine phases $($ Pre2, Pre1, Ictal, Post1, and Interictal). ${ }^{*}, P<0.05 ;{ }^{* *}$, S1, primary somatosensory cortex; Pre2, 24-48 h before headache onset; Pre1, within 24 h before headache onset; Ictal, during migraine attack; Post1, within $24 \mathrm{~h}$ after headache offset; Interictal, the period' $48 \mathrm{~h}$ between the last and next headache phase

\section{Somatosensory gating profile during migraine cycle}

The potentials evoked through paired-pulse stimulation are visualised in Fig. 7 for patient 1 during the interictal period. Prominent first and second somatosensory responses were elicited and peaked at $18 \mathrm{~ms}$ (Fig. 7A). The corresponding topography at $18 \mathrm{~ms}$ indicated contralateral somatosensory activation (Fig. 7B); moreover, the current intensity of the contralateral S1 peaked at 15-20 ms (Fig. 7C). The somatosensory inhibitory capability was determined from the gating ratio (Fig. 7D).
The somatosensory gating ratios were compared across the migraine cycle (Fig. 8). The gating ratio was higher on Pre1 days $(1.01 \pm 0.14)$ than on Ictal days $(0.88 \pm 0.11$; $z=2.2, P=0.028)$.

\section{Discussion}

This study was the first to use consecutive daily EEG recordings for 30 days to elucidate the electrophysiological brainstem and S1 dynamics in terms of the neuronal excitability and inhibition during migraine 


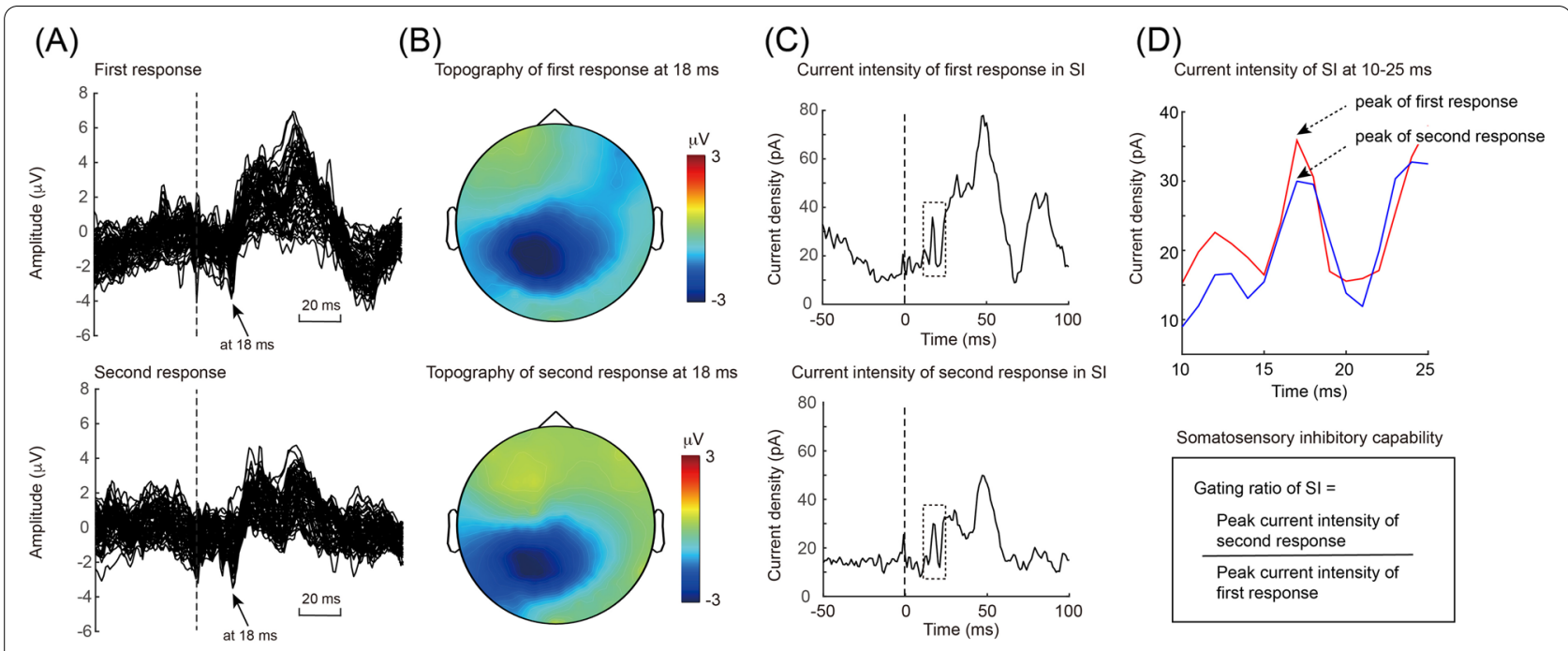

Fig. 7 SSEPs for paired-pulse stimulation. A Superimposed SSEPs (first and second responses) peaked at $18 \mathrm{~ms}$ in response to paired electrical stimulation. B Topographies of peak first and second responses. C S1 current intensity over time for first and second responses. Dashed square indicates peak S1 component. D Acquisition and calculation of S1 gating ratio (inhibitory capability). S1, primary somatosensory cortex

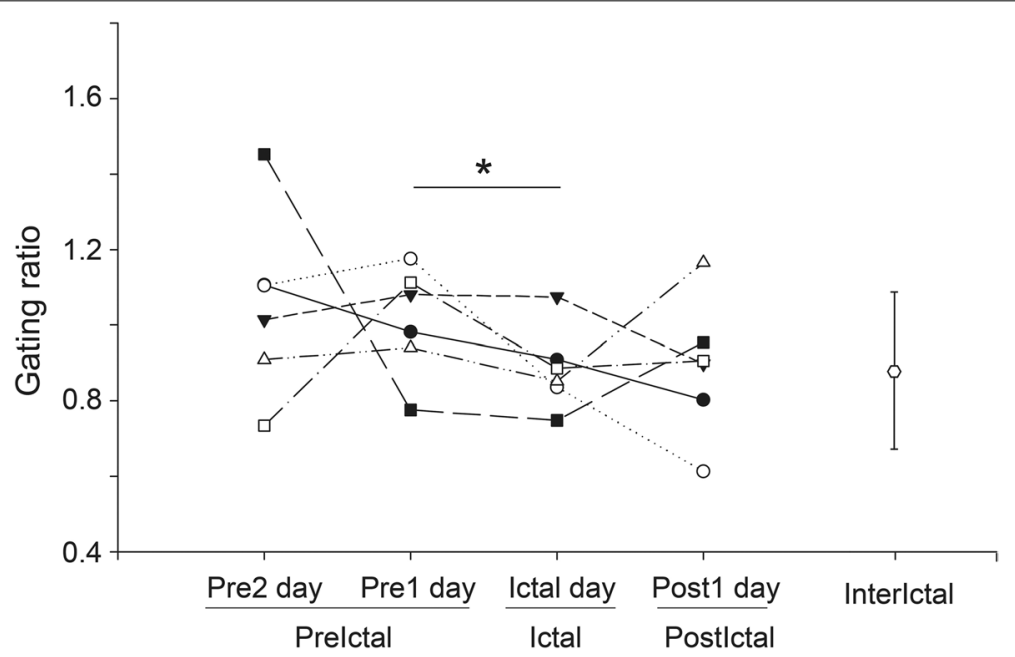

Fig. 8 S1 inhibition during migraine cycle. Differences in S1 gating ratio among migraine cycles $(n=6)$ and migraine phases (Pre2, Pre1, Ictal, Post1, and Interictal). *, $P<0.05$; S1, primary somatosensory cortex; Pre2, 24-48 h before headache onset; Pre1, within $24 \mathrm{~h}$ before headache onset; Ictal, during migraine attack; Post 1 , within $24 \mathrm{~h}$ after headache offset

cycles in patients with EM. The primary finding of this study is that the somatosensory excitability in both the brainstem (peaking at $12-14 \mathrm{~ms}$ after stimulus) and S1 (peaking at $18-19 \mathrm{~ms}$ after stimulus) reached its maxima on the day prior to the acute migraine attack (Pre1) and then decreased; the S1 inhibitory capability was lower in the Pre1 than in the Ictal phase.

\section{Dynamic brain activation during the migraine cycle}

During the migraine cycles, brainstem activation peaked level within $24 \mathrm{~h}$ before headache onset and subsequently declined during the migraine attacks. The brainstem activation in these two phases was also higher than it was during the interictal period. Neuroimaging findings have indicated the cyclic changes in 
brain activity during different phases of the migraine cycle, corroborating our observations of fluctuating activity [8, 11-13, 31-33]. Increased brainstem activation preceding the migraine attack has been observed after trigeminal nociceptive stimulation [33], after noxious orofacial stimulation [34], and in the resting state [31] and is consistent with enhanced resting-state functional connectivity among brainstem nuclei or between brainstem and cortical regions $[13,31]$. In a diffusion tensor imaging study, mean brainstem diffusivity increased during the interictal period, but decreased prominently $24 \mathrm{~h}$ before an attack [35]. Moreover, greater brainstem activity during spontaneous or triggered migraine attacks than during interictal periods has been reported [36-39]. Using nonpainful somatosensory stimulation and EEG, this study verified the alterations in brainstem activation across the migraine cycle, which imply the dysfunctional brainstem functions immediately before and during an attack.

S1 excitability also fluctuated; in particular, S1 activation increased before attacks. This fluctuation in cortical excitability in migraine has been evidenced through amplitude measurements, power spectra, and habituation of contingent negative variation responses [40], resting-state EEG power and coherence [5], and beta event-related desynchronisation during sensorimotor tasks [41, 42]. Nonetheless, in MRI studies, functional connectivity and anisotropy of the thalamus also increases immediately before an attack [31, 35], but its interictal connectivity with the cortical networks [43] is disrupted during an attack [44]. These results suggest that the increased susceptibility to the neuropathological process before an attack might involve a facilitatory thalamocortical mechanism. Therefore, in patients with migraine, the somatosensory network may dynamically amplify afferent traffic or be cyclically hyperresponsive to peripheral stimuli that increase afferent traffic. Consistent with prior hypotheses [3, 38], dysregulation of the central excitability of the somatosensory system could play a primary role in migraine pathophysiology. Furthermore, the cyclic peak latency of evoked responses on Pre1 days occurred earlier in the brainstem (12-14 ms) than it did in the S1 (18-19 ms), indicating that the major driver of migraine pathophysiology might originate in somatosensory neurotransmission in deep brain structures (possibly the brainstem), and S1 activation seems to be reciprocal to the cyclic changes in brainstem activation. In longitudinal 30-day fMRI studies, the relationship between brainstem and $\mathrm{S} 1$ activation during migraine phases has remained undetermined because of the trade-off between the spatial resolution and scanning regions in fMRI techniques.

\section{S1 inhibitory function during migraine cycle}

We discovered that somatosensory gating capability (which is related to habituation) decreased before migraine attacks, a finding consistent with the identification of a preictal maximum habituation deficit [7], suggesting a pre-ictal increase of cortical excitability. The exact pathophysiological mechanism remains unclear, but the preictal dynamics of cortical sensorimotor inhibition have been associated with changes in cortical or thalamic interneuronal activity $[41,45]$. The thalamocortical network is thus implicated in migraine pathophysiology [46], which may indicate that the cyclic S1 habituation and neural synchronisation of the somatosensory network are modulated to some degree by the thalamocortical neurotransmitter system. Moreover, consistent with previous findings [6], we observed no difference in S1 inhibitory function between the interictal and ictal phases in our patients with EM.

\section{Relevance for migraine pathophysiology}

Following several years in which the brainstem was considered the 'migraine generator', attention has recently shifted to the 'hypothalamus. This is because sequential fMRI studies as a response to trigeminal nociceptive stimulation on the same patient have demonstrated that the hypothalamus is activated $24-48 \mathrm{~h}$ before headache onset, and increases its functional connectivity with the brainstem [11, 12]. Our results indicate that the S1 and brainstem both reach maximum activation $24 \mathrm{~h}$ before an attack. The timing of activation is similar to that revealed in the fMRI analysis. In previous neurophysiology studies, researchers have observed that the closer the onset of an attack is, the more the evoked activity of the parietal cortex decreases, eventually returning to normal during an attack [47]. Moreover, in patients with migraine, defects in descending modulatory circuits may contribute to the migraine attack [48]. No other study has verified S1 activity during the pre-attack period (i.e. $48 \mathrm{~h}$ before the onset of the pain phase). Overall, these data, together with the study results, suggest that somatosensory cortex activity plays a key role in the recurrence of migraine attacks.

\section{Limitations}

This study has several limitations. First, limited by the spatial resolution of EEG, this study failed to elucidate the functional roles of specific nuclei, such as the trigeminal nuclei or dorsal pons, in the brainstem during the migraine cycle. Second, although in neuroimaging studies the hypothalamus activation is regarded as a neurological signature of migraine generation [11]; however, we did not observe hypothalamic activation in our EEG analysis. This could be attributed either to the 
insufficiency of the low signal-to-noise ratio for obtaining reliable hypothalamus activation data because of the limited trial number of SSEP responses, or simply because the innocuous somatosensory pathway does not have a hypothalamic relay station [24]. Third, the effects of psychological distress on the cyclic brainstem and S1 activation remain elusive. Fourth, one of our patients was diagnosed as having TTH; this might have confounded the findings. Although several evidences have indicated common cortical disinhibition between migraine and TTH [49] as well as a normal amplitude of visual evoked responses [50], brainstem auditory evoked potentials [51] and contingent negative variation [52] in patients with TTH, the effects of TTH on the cyclical brainstem and SI activation must be identified. Finally, our data were obtained from only two patients; further investigations using larger sample sizes are warranted.

\section{Conclusion}

This study demonstrated that the migraineur's brain is subject to cyclical changes in brainstem and S1 excitability, and the neural substrates involved in the underlying pathophysiological mechanism include both excitation and inhibition. In terms of future migraine treatment, stabilization of brain excitability may be the pivotal feature, and the key target for action may lie in deep brain structures (especially the brainstem). Further investigations with larger sample sizes are warranted.

\section{Abbreviations \\ EM: Episodic migraine; S1: Primary somatosensory cortex; MNE: Minimum norm estimate; SSEPs: Somatosensory-evoked potentials.}

\section{Acknowledgements}

We would like to thank the study participants for actively participating. This work was supported by the Brain Research Center, National Yang Ming Chiao Tung University, from the Featured Areas Research Center Program within the framework of the Higher Education Sprout Project by the Ministry of Education of Taiwan.

\section{Authors' contributions}

F.J. Hsiao, W.T. Chen, and S.J. Wang contributed to the study design. F.J. Hsiao, W.T. Chen, and S.J. Wang recruited patients and collected data. F.J. Hsiao, W.T. Chen, L.L.H. Pan, H.Y. Liu, Y.F. Wang, S.P. Chen, K.L. Lai, and S.J. Wang performed data analysis and interpretation. F.J. Hsiao, W.T. Chen, and G. Coppola wrote the manuscript. W.T. Chen, G. Coppola, and S.J. Wang critically reviewed the article. All authors interpreted the data, reviewed the manuscript, and approved the final version.

\section{Funding}

This work was funded by the Ministry of Science and Technology of Taiwan (MOST 109-2314-B-075 -050 -MY2 to WT Chen, 108-2321-B-010-001, 108-2321-B-010-014-MY2, 110-2321-B-010-005, and 111-2321-B-010-004 to SJ Wang, and 108-2221-E-010-004 and 109-2221-E-003-MY2 to FJ Hsiao) and Taipei Veterans General Hospital (V108C-129 and V107C-091 to WT Chen).

\section{Availability of data and materials}

The datasets generated during and/or analyzed during the current study are available from the corresponding authors on reasonable request.

\section{Declarations}

\section{Ethics approval and consent to participate}

Participants provided signed informed consent. The hospital's institutional review board approved the study protocol (VGHTPE: IRB 2019-07-001B).

\section{Competing interests}

FJ Hsiao, WT Chen, LL Pan, HY Liu, YF Wang, SP Chen and KL Lai declare no potential conflicts of interest. G Coppola reports travel fees from Novartis Italy, Eli Lilly, and TEVA, all outside the submitted work. SJ Wang reports grants and personal fees from Novartis Taiwan, personal fees from Daiichi-Sankyo, grants and personal fees from Eli-Lilly, personal fees from Allergan, personal fees from Pfizer, Taiwan, personal fees from Bayer, Taiwan, outside the submitted work.

\section{Author details}

${ }^{1}$ Brain Research Center, National Yang Ming Chiao Tung University, Taipei, Taiwan. ${ }^{2}$ School of Medicine, National Yang Ming Chiao Tung University, Taipei, Taiwan. ${ }^{3}$ Department of Neurology, Neurological Institute, Taipei Veterans General Hospital, 201, Shihpai Rd Sec 2, Taipei 112, Taiwan. ${ }^{4}$ Department of Neurology, Keelung Hospital, Ministry of Health and Welfare, Keelung, Taiwan. ${ }^{5}$ Department of Medico-Surgical Sciences and Biotechnologies, Sapienza University of Rome Polo Pontino, Latina, Italy.

Received: 12 December 2021 Accepted: 20 January 2022

Published online: 05 February 2022

\section{References}

1. Disease GBD, Injury I, Prevalence C (2017) Global, regional, and national incidence, prevalence, and years lived with disability for 328 diseases and injuries for 195 countries, 1990-2016: a systematic analysis for the global burden of Disease study 2016. Lancet 390:1211-1259

2. Burstein R, Noseda R, Borsook D (2015) Migraine: multiple processes, complex pathophysiology. J Neurosci 35:6619-6629

3. Dodick DW (2018) Migraine. Lancet 391:1315-1330

4. de Tommaso M, Ambrosini A, Brighina F, Coppola G, Perrotta A, Pierelli F, Sandrini G, Valeriani M, Marinazzo D, Stramaglia S, Schoenen J (2014) Altered processing of sensory stimuli in patients with migraine. Nat Rev Neurol 10:144-155

5. Cao Z, Lin CT, Chuang CH, Lai KL, Yang AC, Fuh JL, Wang SJ (2016) Resting-state EEG power and coherence vary between migraine phases. J Headache Pain 17:102

6. Chen WT, Hsiao FJ, Ko YC, Liu HY, Wang PN, Fuh JL, Lin YY, Wang SJ (2018) Comparison of somatosensory cortex excitability between migraine and "strict-criteria" tension-type headache: a magnetoencephalographic study. Pain 159:793-803

7. Chen WT, Wang SJ, Fuh JL, Lin CP, Ko YC, Lin YY (2009) Peri-ictal normalization of visual cortex excitability in migraine: an MEG study. Cephalalgia 29:1202-1211

8. Stankewitz A, May A (2007) Cortical excitability and migraine. Cephalalgia 27:1454-1456

9. Stankewitz A, May A (2009) The phenomenon of changes in cortical excitability in migraine is not migraine-specific--a unifying thesis. Pain 145:14-17

10. Siniatchkin M, Kropp P, Gerber WD, Stephani U (2000) Migraine in childhood--are periodically occurring migraine attacks related to dynamic changes of cortical information processing? Neurosci Lett 279:1-4

11. Schulte LH, May A (2016) The migraine generator revisited: continuous scanning of the migraine cycle over 30 days and three spontaneous attacks. Brain 139:1987-1993

12. Schulte LH, Mehnert J, May A (2020) Longitudinal neuroimaging over 30 days: temporal characteristics of migraine. Ann Neurol 87:646-651

13. Schulte LH, Menz MM, Haaker J, May A (2020) The migraineur's brain networks: continuous resting state fMRI over 30 days. Cephalalgia 40:1614-1621

14. Akerman S, Holland PR, Goadsby PJ (2011) Diencephalic and brainstem mechanisms in migraine. Nat Rev Neurosci 12:570-584

15. Attwell D, ladecola C (2002) The neural basis of functional brain imaging signals. Trends Neurosci 25:621-625 
16. Logothetis NK (2008) What we can do and what we cannot do with fMRI. Nature 453:869-878

17. Iannetti GD, Mouraux A (2010) From the neuromatrix to the pain matrix (and back). Exp Brain Res 205:1-12

18. Porcaro C, Di Lorenzo G, Seri S, Pierelli F, Tecchio F, Coppola G (2017) Impaired brainstem and thalamic high-frequency oscillatory EEG activity in migraine between attacks. Cephalalgia 37:915-926

19. Coppola G, Di Lorenzo C, Schoenen J, Pierelli F (2013) Habituation and sensitization in primary headaches. J Headache Pain 14:65

20. Hsiao FJ, Wang SJ, Lin YY, Fuh JL, Ko YC, Wang PN, Chen WT (2018) Somatosensory gating is altered and associated with migraine chronification: a magnetoencephalographic study. Cephalalgia 38:744-753

21. (2018) Headache Classification Committee of the International Headache Society (IHS) The International Classification of Headache Disorders, 3rd edition. Cephalalgia 38:1-211

22. Ling YH, Chen SP, Fann CS, Wang SJ, Wang YF (2019) TRPM8 genetic variant is associated with chronic migraine and allodynia. J Headache Pain 20:115

23. Oostenveld R, Praamstra P (2001) The five percent electrode system for high-resolution EEG and ERP measurements. Clin Neurophysiol 112:713-719

24. Attal Y, Bhattacharjee M, Yelnik J, Cottereau B, Lefevre J, Okada Y, Bardinet E, Chupin M, Baillet S (2007) Modeling and detecting deep brain activity with MEG \& EEG. Annu Int Conf IEEE Eng Med Biol Soc 2007:4937-4940

25. Hsiao FJ, Cheng CH, Chen WT, Lin YY (2013) Neural correlates of somatosensory paired-pulse suppression: a MEG study using distributed source modeling and dynamic spectral power analysis. Neuroimage 72:133-142

26. Hsiao FJ, Wang SJ, Lin YY, Fuh JL, Ko YC, Wang PN, Chen WT (2017) Altered insula-default mode network connectivity in fibromyalgia: a resting-state magnetoencephalographic study. J Headache Pain 18:89

27. Hamalainen MS, IImoniemi RJ (1994) Interpreting magnetic fields of the brain: minimum norm estimates. Med Biol Eng Comput 32:35-42

28. Attal Y, Schwartz D (2013) Assessment of subcortical source localization using deep brain activity imaging model with minimum norm operators: a MEG study. PLoS One 8:e59856

29. Gramfort A, Papadopoulo T, Olivi E, Clerc M (2010) OpenMEEG: opensource software for quasistatic bioelectromagnetics. Biomed Eng Online 9:45

30. Tadel F, Baillet S, Mosher JC, Pantazis D, Leahy RM (2011) Brainstorm: a user-friendly application for MEG/EEG analysis. Comput Intell Neurosci 2011:879716

31. Meylakh N, Marciszewski KK, Di Pietro F, Macefield VG, Macey PM, Henderson LA (2018) Deep in the brain: changes in subcortical function immediately preceding a migraine attack. Hum Brain Mapp 39:2651-2663

32. Meylakh N, Marciszewski KK, Di Pietro F, Macefield VG, Macey PM, Henderson LA (2021) Brainstem functional oscillations across the migraine cycle: a longitudinal investigation. Neurolmage Clinical 30:102630

33. Stankewitz A, Aderjan D, Eippert F, May A (2011) Trigeminal nociceptive transmission in migraineurs predicts migraine attacks. J Neurosci 31:1937-1943

34. Marciszewski KK, Meylakh N, Di Pietro F, Mills EP, Macefield VG, Macey PM, Henderson LA (2018) Changes in brainstem pain modulation circuitry function over the migraine cycle. J Neurosci 38:10479-10488

35. Marciszewski KK, Meylakh N, Di Pietro F, Macefield VG, Macey PM, Henderson LA (2019) Fluctuating regional brainstem diffusion imaging measures of microstructure across the migraine cycle. eNeuro 6:ENEURO.0005-19.2019.

36. Afridi SK, Giffin NJ, Kaube H, Friston KJ, Ward NS, Frackowiak RS, Goadsby PJ (2005) A positron emission tomographic study in spontaneous migraine. Arch Neurol 62:1270-1275

37. Afridi SK, Matharu MS, Lee L, Kaube H, Friston KJ, Frackowiak RS, Goadsby PJ (2005) A PET study exploring the laterality of brainstem activation in migraine using glyceryl trinitrate. Brain 128:932-939

38. Bahra A, Matharu MS, Buchel C, Frackowiak RS, Goadsby PJ (2001) Brainstem activation specific to migraine headache. Lancet 357:1016-1017

39. Weiller C, May A, Limmroth V, Juptner M, Kaube H, Schayck RV, Coenen HH, Diener HC (1995) Brain stem activation in spontaneous human migraine attacks. Nat Med 1:658-660
40. Siniatchkin M, Gerber WD, Kropp P, Vein A (1999) How the brain anticipates an attack: a study of neurophysiological periodicity in migraine. Funct Neurol 14:69-77

41. Mykland MS, Bjork MH, Stjern M, Omland PM, Uglem M, Sand T (2019) Fluctuations of sensorimotor processing in migraine: a controlled longitudinal study of beta event related desynchronization. J Headache Pain 20:77

42. Mykland MS, Bjork MH, Stjern M, Sand T (2018) Alterations in post-movement beta event related synchronization throughout the migraine cycle: a controlled, longitudinal study. Cephalalgia 38:718-729

43. Coppola G, Di Renzo A, Tinelli E, Lepre C, Di Lorenzo C, Di Lorenzo G, Scapeccia M, Parisi V, Serrao M, Colonnese C, Schoenen J, Pierelli F (2016) Thalamo-cortical network activity between migraine attacks: insights from MRI-based microstructural and functional resting-state network correlation analysis. J Headache Pain 17:100

44. Coppola G, Di Renzo A, Tinelli E, Di Lorenzo C, Di Lorenzo G, Parisi V, Serrao M, Schoenen J, Pierelli F (2016) Thalamo-cortical network activity during spontaneous migraine attacks. Neurology 87:2154-2160

45. Coppola G, Di Lenola D, Abagnale C, Ferrandes F, Sebastianelli G, Casillo F, Di Lorenzo C, Serrao M, Evangelista M, Schoenen J, Pierelli F (2020) Shortlatency afferent inhibition and somato-sensory evoked potentials during the migraine cycle: surrogate markers of a cycling cholinergic thalamocortical drive? J Headache Pain 21:34

46. Coppola G, De Pasqua V, Pierelli F, Schoenen J (2012) Effects of repetitive transcranial magnetic stimulation on somatosensory evoked potentials and high frequency oscillations in migraine. Cephalalgia 32:700-709

47. Coppola G, Bracaglia M, Di Lenola D, lacovelli E, Di Lorenzo C, Serrao M, Evangelista M, Parisi V, Schoenen J, Pierelli F (2016) Lateral inhibition in the somatosensory cortex during and between migraine without aura attacks: correlations with thalamocortical activity and clinical features. Cephalalgia 36:568-578

48. Russo A, Tessitore A, Esposito F, Marcuccio L, Giordano A, Conforti R, Truini A, Paccone A, d'Onofrio F, Tedeschi G (2012) Pain processing in patients with migraine: an event-related fMRI study during trigeminal nociceptive stimulation. J Neurol 259:1903-1912

49. de Tommaso M, Sciruicchio V, Guido M, Sasanelli G, Puca F (1999) Steadystate visual-evoked potentials in headache: diagnostic value in migraine and tension-type headache patients. Cephalalgia 19:23-26 discussion 1

50. Wang W, Wang GP, Ding XL, Wang YH (1999) Personality and response to repeated visual stimulation in migraine and tension-type headaches. Cephalalgia 19:718-724 discussion 697-8

51. Unay B, Ulas UH, Karaoglu B, Eroglu E, Akin R, Gokcay E (2008) Visual and brainstem auditory evoked potentials in children with headache. Pediatr Int 50:620-623

52. Maertens de Noordhout A, Timsit-Berthier M, Timsit M, Schoenen J (1986) Contingent negative variation in headache. Ann Neurol 19:78-80

\section{Publisher's Note}

Springer Nature remains neutral with regard to jurisdictional claims in published maps and institutional affiliations.

Ready to submit your research? Choose BMC and benefit from:

- fast, convenient online submission

- thorough peer review by experienced researchers in your field

- rapid publication on acceptance

- support for research data, including large and complex data types

- gold Open Access which fosters wider collaboration and increased citations

- maximum visibility for your research: over 100M website views per year

At BMC, research is always in progress.

Learn more biomedcentral.com/submissions 